\title{
Chemoimmunotherapy with brentuximab vedotin combined with ifosfamide, gemcitabine, and vinorelbine is highly active in relapsed or refractory classical Hodgkin lymphoma
}

\author{
Khadega A. Abuelgasim ${ }^{1,2,3} \cdot$ Mohsen Alzahrani $^{1,2} \cdot$ Yousef Alsharhan $^{3} \cdot$ Moataz Khairi $^{3} \cdot$ Mohammed Hommady $^{3}$.

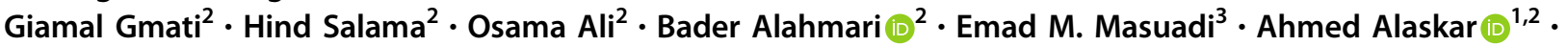 \\ Ayman Alhejazi $^{2} \cdot$ Moussab Damlaj $^{1,2,3}$
}

Received: 13 August 2018 / Revised: 18 December 2018 / Accepted: 7 January 2019 / Published online: 30 January 2019

(c) The Author(s) 2019. This article is published with open access

Salvage therapy followed by autologous hematopoietic stem cell transplantation (HCT) can be curative in relapsed/ refractory classical Hodgkin lymphoma (cHL) [1]. Complete response $(\mathrm{CR})$ prior to HCT, particularly complete metabolic response (CMR) indicated by a negative positron emission tomography/computed tomography (PET/CT) scan, is highly prognostic of post-transplant outcome [2, 3]. Thus optimization of disease status prior to HCT is highly desirable. There is no gold standard salvage in $\mathrm{R} / \mathrm{R} \mathrm{cHL}$ prior to HCT, and the choice of regimen depends on the physicians' experience and preference. Brentuximab vedotin $(\mathrm{Bv})$ has demonstrated excellent activity in cHL. Recent reports have combined $\mathrm{Bv}$ with standard salvage or with PD-1 inhibitor therapy with higher responses than conventional chemotherapy alone [4-6]. Our aim from this analysis is to examine the efficacy of $\mathrm{Bv}$ incorporated within the gemcitabine salvage regimen ifosfamide, gemcitabine, and vinorelbine (IGEV-Bv).

After institutional review board (IRB) approval, patients' $\geq 14$ years of age with relapsed or refractory cHL who received IGEV-Bv at our institution between 2013 and 2017 were identified, and all records were retrospectively extracted. Patients were eligible if they had histologically proven evidence of disease and those who achieved a partial

Moussab Damlaj

damlajmo@ngha.med.sa

1 King Abdulla International Medical Research Center, Riyadh, Saudi Arabia

2 King Abdulaziz Medical City, Department of Oncology, Riyadh, Saudi Arabia

3 King Saud bin Abdulaziz University for Health Sciences, Riyadh, Saudi Arabia metabolic response (PMR) or better to salvage therapy proceeded to HCT [7]. Patients received IGEV as first or subsequent salvage (FS or SS), as previously described [8]. $\mathrm{Bv}$ was administered at a dose of $1.8 \mathrm{mg} / \mathrm{kg}$ body weight on day 1 of each 3 -week IGEV course. A minimum of two cycles of salvage were administered to all patients, and those who did not attain at least a PMR status following two cycles of salvage were switched to an alternate non-cross resistant regimen.

All analyzed patients underwent PET/CT staging following one or two cycles of IGEV-Bv to assess response. All studies were performed on GE 710 discovery TF system. Standardized uptake value of the liver and mediastinum is noted, and update was classified per Deauville criteria as $\leq$ liver uptake or $\leq$ mediastinal blood pool [9]. Patients with uptake $\leq$ liver (i.e., Deauville 3) were deemed to have CMR. Patients received BEAM (carmustine, etoposide, cytarabine, and melphalan) as conditioning followed by autologous stem cell rescue. All patients were hospitalized during conditioning therapy and until platelet and neutrophil engraftment.

Overall survival (OS) was calculated from the date of stem cell infusion until the date of death of any cause or last documented follow-up. Progression-free survival (PFS) was calculated from the date of stem cell infusion until death of any cause or evidence of disease progression or relapse. Baseline patient, disease, and treatment-related variables were reported using descriptive statistics (counts, medians, and percentages). Probability of OS and PFS was computed using the Kaplan-Meier method. Group comparisons were made using the log-rank test. Statistical analyses were performed using JMP Pro Version 11 (SAS Institute, Cary, NC, USA) software and EZR on R commander.

A total of 28 patients met the eligibility criteria and were included in this analysis. The median age was 25 (15-49) 
Table 1 Baseline characteristics and therapy of the cohort

\begin{tabular}{|c|c|}
\hline Characteristics & Entire cohort $(n=28)$ \\
\hline Male, $n(\%)$ & $15(53 \%)$ \\
\hline Age at $\mathrm{HCT}$, median (range) & $25(15-49)$ \\
\hline \multicolumn{2}{|l|}{ Stage at Dx, $n(\%)$} \\
\hline II & $7(25)$ \\
\hline III & $6(21)$ \\
\hline IV & $15(54)$ \\
\hline Constitutional Symptoms at Dx, $n(\%)$ & $20(71)$ \\
\hline Bulky Disease at Dx, $n(\%)$ & $8(29)$ \\
\hline \multicolumn{2}{|l|}{ Front Line Treatment, $n(\%)$} \\
\hline$A B V D$ & $23(82)$ \\
\hline $\mathrm{ABVD} \rightarrow$ EscBEACOPP & $4(14)$ \\
\hline Other & $1(4)$ \\
\hline IFRT, $n(\%)$ & $5(18)$ \\
\hline Refractory ( $\leq 3$ months remission), $n(\%)$ & $12(43)$ \\
\hline Time to relapse, median months (range) & $7.9(1.9-133)$ \\
\hline \multicolumn{2}{|l|}{ IGEV-Bv order of salvage, $n(\%)$} \\
\hline First & $14(50)$ \\
\hline Subsequent & $14(50)$ \\
\hline No. of salvage cycles, median (range) & $2(2-6)$ \\
\hline $\begin{array}{l}\text { Number of cycles at stem cell collection, } \\
\text { median (range) }\end{array}$ & $2(1-5)$ \\
\hline Days of collection, median (range) & $1(1-2)$ \\
\hline $\mathrm{CD} 34 \times 10^{6} / \mathrm{kg}$ collected, median (range) & $13.6(2.8-44.8)$ \\
\hline \multicolumn{2}{|l|}{ PET/CT status post IGEV-Bv, $n(\%)$} \\
\hline Complete metabolic response & $20(71)$ \\
\hline Partial metabolic response & $7(25)$ \\
\hline Stable disease & $1(4)$ \\
\hline Median follow-up, months (range) & $17(0-65)$ \\
\hline
\end{tabular}

$H C T$ hematopoietic stem cell transplant, $D x$ diagnosis, PET/CT positron emission tomography/computed tomography, $A B V D$ doxorubicin, bleomycin, vinblastine, and dacarbazine, escBEACOPP escalated bleomycin, etoposide, doxorubicin, cyclophosphamide, vincristine, procarbazine, and prednisone, IFRT involved field radiotherapy, $I G E V-B v$ ifosfamide, gemcitabine, and vinorelbine with brentuximab vedotin

years, and 15 (53\%) were men. All patients had early unfavorable or advanced stage disease at diagnosis with 8 (29\%) having a bulky mass and $20(71 \%)$ with constitutional symptoms. A total of $12(43 \%)$ patients had refractory disease with evidence of progression within 3 months following completion of front-line therapy. All patients achieved at least a PMR and were able to proceed to HCT. Response assessment after IGEV-Bv (one or two cycles) showed CMR in $20(71 \%)$ and PMR in $7(25 \%)$ and stable disease in $1(4 \%)$. Baseline characteristics and response are shown in Table 1. The most common toxicities observed were hematologic with grades 3-4 neutropenia and thrombocytopenia at $96 \%$ and $89 \%$. Median number of units of red blood cells transfused during the course of salvage
Table 2 Observed adverse events following IGEV-Bv salvage and Bv consolidation

\begin{tabular}{ll}
\hline Adverse event & Entire cohort $(n=28)$ \\
\hline Neutropenia grades 3-4, $n(\%)$ & $27(96)$ \\
Thrombocytopenia grades 3-4, $n(\%)$ & $25(89)$ \\
Blood transfusion units, median (range) & $2(0-11)$ \\
Mucositis, $n$ (\%) & \\
$\quad$ Grades 1-2 & $5(18)$ \\
$\quad$ Grade 3 & $1(4)$ \\
Febrile neutropenia, $n(\%)$ & $16(57)$ \\
Peripheral neuropathy on salvage, $n(\%)$ & \\
$\quad$ Grades 1-2 & $2(7)$ \\
$\quad$ Grade 3 & $1(4)$ \\
Diarrhea, $n$ (\%) & $6(21)$ \\
Transaminitis grades 3-4, $n(\%)$ & $2(7)$ \\
Acute renal injury, $n(\%)$ & 0 \\
ICU transfer, $n$ (\%) & 0 \\
Bv consolidation, $n$ (\%) & $18(64)$ \\
Indication for Bv consolidation, $n(\%)$ & \\
$\quad$ Remission $<12$ Months & $13(72)$ \\
$\quad$ B-symptoms at Relapse & $3(17)$ \\
$\quad$ Extranodal Relapse & $2(11)$ \\
Total doses of Bv delivered, median (range) & $15(6-16)$ \\
Filgrastim given during Bv consolidation, $n(\%)$ & $7(39)$ \\
Peripheral neuropathy on consolidation, $n(\%)$ & \\
Grades 1-2 & $4(22)$ \\
Grade 3 & $1(5)$ \\
Bv dose reduced due to AE, $n(\%)$ & $6(33)$ \\
\hline
\end{tabular}

$H C T$ hematopoietic stem cell transplant, $I C U$ intensive care unit, $I G E V-B v$ ifosfamide, gemcitabine, and vinorelbine with brentuximab vedotin, $A E$ adverse events

therapy was 2. Febrile neutropenia was seen in 16 (57\%). No transfer to the intensive care unit or mortality during salvage chemotherapy. Other observed adverse events (AE) are shown in Table 2.

Median (range) of CD34 collected cells were 13.6 $(2.8-44.8) \times 10^{6} / \mathrm{kg}$ after a median of two cycles of salvage chemotherapy. Consolidative $\mathrm{Bv}$ post HCT was given to 18 (64\%) mainly due to relapsed disease within 12 months following front-line therapy with a median (range) of 15 (6-16) doses administered pre- and postHCT. Dose adjustment in Bv was done in $6(33 \%)$ due to $\mathrm{AE}$ predominantly neutropenia or neuropathy as shown in Table 2.

Post HCT, the estimated 2-year PFS and 2-year OS were $87.1 \%$ (65-95.7\%) and 73.5\% (49.8-87.3), respectively. A total of six patients experienced disease relapse post HCT and three patients died; due to progressive disease in two and pulmonary infection in one. IGEV-Bv as FS vs. SS resulted in a superior PFS and trend toward improved OS at 100 vs. $75 \%(40.8-91.2) p=0.0078$ and 100 vs. $50 \%$ (20.8-73.6) $p=0.08$, respectively. These results are shown in Fig. 1.

Autologous HCT is a potentially curative therapy in R/R cHL with approximately half of patients achieving 
a

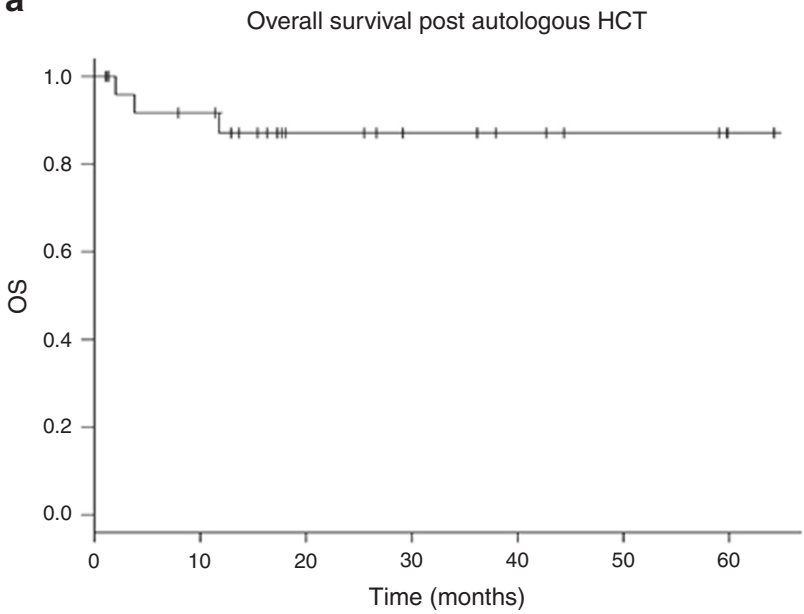

C

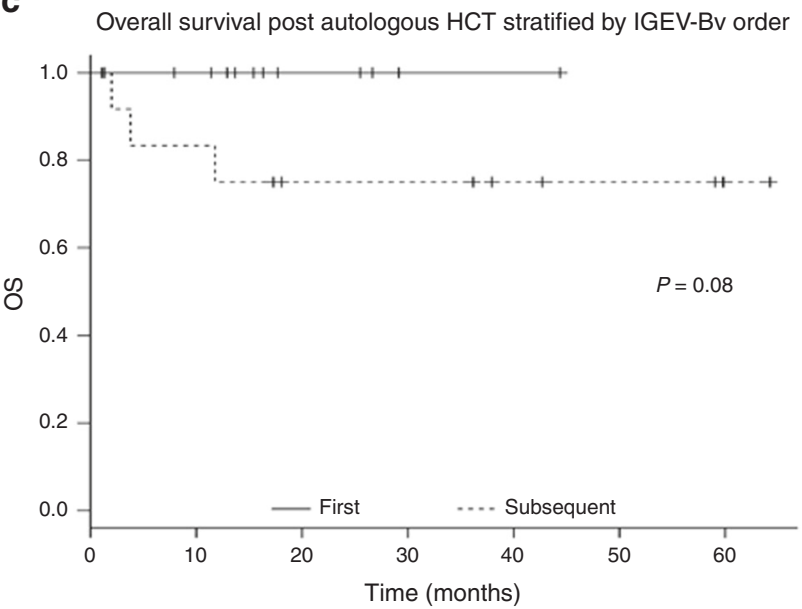

b

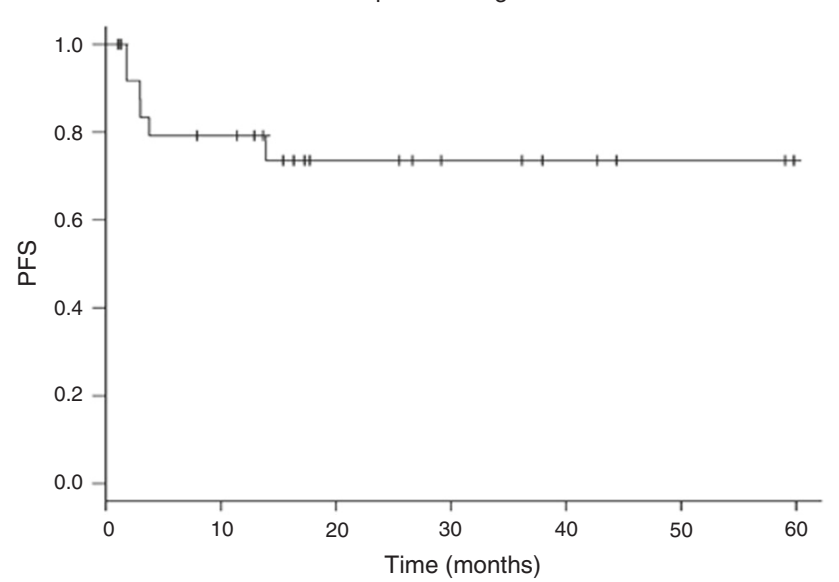

d

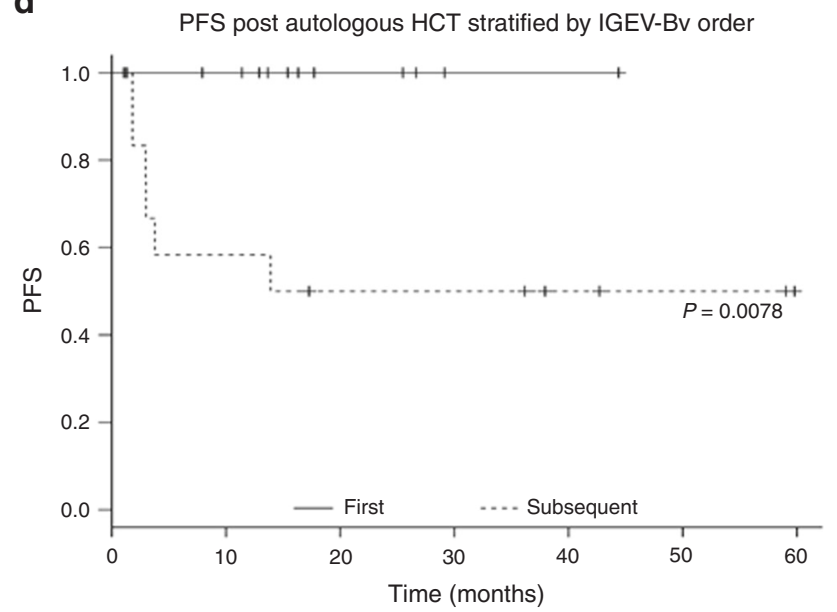

Fig. 1 Progression-free survival (PFS) and overall survival (OS) post autologous HCT. a OS for the whole cohort; b PFS for the whole cohort; c Overall survival stratified by IGEV-Bv salvage order; $\mathbf{d}$ PFS stratified by IGEV-Bv salvage order

prolonged remissions following standard salvage therapy followed by HCT [10]. Importantly, CMR status pre-HCT indicated by a negative PET/CT has been shown to strongly correlate with a superior outcome by a number of groups [2, 3]. Thus, optimization of disease status prior to HCT leads to higher remission rates. In this study, we observed that addition of standard dose Bv to IGEV salvage was associated with a $70 \% \mathrm{CMR}$ rate leading to favorable post-HCT outcome in this high-risk cohort of patients. Importantly, $\sim 50 \%$ of the cohort received IGEV as SS after failing to achieve PMR with previous salvage and fared relatively favorably than expected. Previously, Villa et al. reported that patients requiring a second salvage to attain disease control prior to HCT have a poor outcome with an estimated 5-year PFS and 5-year OS of only 11 and $20 \%$, respectively [11].

The choice of salvage regimen in $\mathrm{R} / \mathrm{R}$ cHL patients eligible for HCT is unknown and clinical practice varies among centers. Although no prospective comparisons of salvage regimens in the setting of $\mathrm{R} / \mathrm{R} \mathrm{cHL}$ were done, they appear to be comparable with regards to efficacy. Santoro et al. treated 91 patients with $\mathrm{R} / \mathrm{R} \mathrm{cHL}$ with $\mathrm{IGEV}$ and observed a relatively high response rates with ORR and CR rates of 81.3 and $53.8 \%$, respectively, with a low-toxicity profile and high-mobilizing potential of stem cells [8]. As the outcome of patients can be optimized with deeper responses prior to $\mathrm{HCT}$, and that $\mathrm{CR}$ status is not achieved in the majority of cases, ongoing efforts to further enhance responses with available regimens are underway.

Chemo-immunotherapy approaches combining Bv with salvage regimens to overcome chemotherapy resistance has been under active investigation. O'Conner et al. reported an international, multicenter phase $1-2$ of $\mathrm{Bv}$ in combination with bendamustine $(\mathrm{Be})$ as an outpatient salvage regimen in $\mathrm{R} / \mathrm{R}$ cHL [12]. Importantly, the recommended dose for the phase 2 of the trial was $1.8 \mathrm{mg} / \mathrm{kg}$ of $\mathrm{Bv}$ and $90 \mathrm{mg} / \mathrm{m}^{2}$ of Be every 3 weeks corresponding to the standard dose of either drug as single agents in clinical practice. However, the proportion of patients achieving CR remains lower than desired and a concern regarding stem cell mobilization 
particularly in elderly patients $>60$ years was observed. $\mathrm{Be}-\mathrm{Bv}$ combination was also recently reported by LaCasce et al. in a group of 55 patients observing a $\mathrm{CR}$ rate of $73.6 \%$ with excellent post-HCT outcome [5]. A total of 31 patients received $\mathrm{Bv}$ monotherapy following $\mathrm{Be}-\mathrm{Bv}$, among them 25 patients received it in the setting of post-HCT consolidation. After a median follow-up of 20.9 months, the estimated 2-year PFS was $69.8 \%$ for those who underwent $\mathrm{HCT}$ and $62.6 \%$ in non-HCT recipients. Other groups examined the incorporation of $\mathrm{Bv}$ within an ESHAP backbone (BRESHAP) in $27 \mathrm{R} / \mathrm{R}$ cHL patients in a phase I/II trial; CR was achieved in 16 out of 17 evaluable patients prior to HCT with no grade III or IV toxicity [4]. More recently, attempts at salvage therapy examined the combination of $\mathrm{Bv}$ along with nivolumab in $\mathrm{R} / \mathrm{R}$ cHL with preliminary results showing an impressive ORR of $82 \%$ with a CR rate of $61 \%$ and only a minority of patients $(<10 \%)$ requiring systemic steroids for immune mediated adverse effects [6].

This analysis has some important limitations, particularly with regards to the sample size and retrospective design. Furthermore, similar to the study by LaCasce et al., some patients received $\mathrm{Bv}$ as consolidation monotherapy post HCT, which may further enhance the post-HCT outcome as previously shown in the AETHERA trial [13]. However, $71 \%$ of patients achieved CMR status pre-HCT, which is perhaps the most important predictor of outcome as shown by multiple groups. Additionally, 27/28 (96\%) of patients were able to proceed to HCT following IGEV-Bv. The follow-up is relatively short, but in a cohort of patients where over $70 \%$ progressed or relapsed within 1 year following front-line therapy, the majority of events are expected to take place within this follow-up time frame in such high-risk patients as shown previously by other studies $[10,13]$. In conclusion, we demonstrate that IGEV-Bv is associated with high response rates even in heavily pretreated patients without compromising stem cell mobilization leading to HCT in the majority of cases. Given the limitations of this analysis, these observations warrant further examination.

\section{Compliance with ethical standards}

Conflict of interest The authors declare that they have no conflict of interest.

Publisher's note: Springer Nature remains neutral with regard to jurisdictional claims in published maps and institutional affiliations.

Open Access This article is licensed under a Creative Commons Attribution 4.0 International License, which permits use, sharing, adaptation, distribution and reproduction in any medium or format, as long as you give appropriate credit to the original author(s) and the source, provide a link to the Creative Commons license, and indicate if changes were made. The images or other third party material in this article are included in the article's Creative Commons license, unless indicated otherwise in a credit line to the material. If material is not included in the article's Creative Commons license and your intended use is not permitted by statutory regulation or exceeds the permitted use, you will need to obtain permission directly from the copyright holder. To view a copy of this license, visit http://creativecommons. org/licenses/by/4.0/.

\section{References}

1. Sureda A, Bader P, Cesaro S, Dreger P, Duarte RF, Dufour C, et al. Indications for allo- and auto-SCT for haematological diseases, solid tumours and immune disorders: current practice in Europe, 2015. Bone Marrow Transplant. 2015;50:1037-56.

2. Smeltzer JP, Cashen AF, Zhang Q, Homb A, Dehdashti F, Abboud $\mathrm{CN}$, et al. Prognostic significance of FDG-PET in relapsed or refractory classical Hodgkin lymphoma treated with standard salvage chemotherapy and autologous stem cell transplantation. Biol Blood Marrow Transplant. 2011;17:1646-52.

3. Damlaj M, Ghazi S, Syed G, Pasha T, Gmati G, Salama H, et al. Pre-autologous transplantation PET/CT using Deauville criteria is an independent predictor of progression in relapsed refractory classical Hodgkin lymphoma. Bone Marrow Transplant. 2017; 52:1342-4.

4. Garcia-Sanz RSA, Alonso-Alvarez S, Gonzalez AP, Rodriguez A, Salar A, et al. Evaluation of the regimen brentuximab vedotin plus ESHAP (BRESHAP) in refractory or relapsed Hodgkin lymphoma patients: preliminary results of a phase I-II trial from the Spanish group of lymphoma and bone marrow transplantation (GELTAMO). Blood. 2015;126:582.

5. LaCasce AS, Bociek RG, Sawas A, Caimi P, Agura E, Matous J, et al. Brentuximab vedotin plus bendamustine: a highly active first salvage regimen for relapsed or refractory Hodgkin lymphoma. Blood. 2018;132:40-8.

6. Herrera AF, Moskowitz AJ, Bartlett NL, Vose JM, Ramchandren $\mathrm{R}$, Feldman TA, et al. Interim results of brentuximab vedotin in combination with nivolumab in patients with relapsed or refractory Hodgkin lymphoma. Blood. 2018;131:1183-94.

7. Cheson BD, Fisher RI, Barrington SF, Cavalli F, Schwartz LH, Zucca E, et al. Recommendations for initial evaluation, staging, and response assessment of Hodgkin and non-Hodgkin lymphoma: the Lugano classification. J Clin Oncol. 2014;32:3059-68.

8. Santoro A, Magagnoli M, Spina M, Pinotti G, Siracusano L, Michieli M, et al. Ifosfamide, gemcitabine, and vinorelbine: a new induction regimen for refractory and relapsed Hodgkin's lymphoma. Haematologica. 2007;92:35-41.

9. Barrington SF, Mikhaeel NG, Kostakoglu L, Meignan M, Hutchings M, Mueller SP, et al. Role of imaging in the staging and response assessment of lymphoma: consensus of the International Conference on Malignant Lymphomas Imaging Working Group. J Clin Oncol. 2014;32:3048-58.

10. Majhail NS, Weisdorf DJ, Defor TE, Miller JS, McGlave PB, Slungaard A, et al. Long-term results of autologous stem cell transplantation for primary refractory or relapsed Hodgkin's lymphoma. Biol Blood Marrow Transplant. 2006;12:1065-72.

11. Villa D, Seshadri T, Puig N, Massey C, Tsang R, Keating A, et al. Second-line salvage chemotherapy for transplant-eligible patients with Hodgkin's lymphoma resistant to platinumcontaining first-line salvage chemotherapy. Haematologica. 2012;97:751-7.

12. O'Connor OA, Lue JK, Sawas A, Amengual JE, Deng C, Kalac M, et al. Brentuximab vedotin plus bendamustine in relapsed or refractory Hodgkin's lymphoma: an international, 
multicentre, single-arm, phase 1-2 trial. Lancet Oncol. 2018; 19:257-66.

13. Moskowitz CH, Nademanee A, Masszi T, Agura E, Holowiecki $\mathrm{J}$, Abidi $\mathrm{MH}$, et al. Brentuximab vedotin as consolidation therapy after autologous stem-cell transplantation in patients with Hodgkin's lymphoma at risk of relapse or progression (AETHERA): a randomised, double-blind, placebo-controlled, phase 3 trial. Lancet. 2015;385:1853-62. 